\title{
Nonlinear Analysis of Guillain Barré Time Series to Elucidate Its Epidemiology
}

\author{
Zurina Lestayo O'Farrill, ${ }^{1}$ José Luís Hernández Cáceres, ${ }^{2}$ and Esperanza O'Farrill Mons ${ }^{3}$ \\ ${ }^{1}$ Neuromuscular Investigation Group, National Institute of Neurology and Neurosurgery, Vedado CP10400, Cuba \\ ${ }^{2}$ Bioinformatics Group, Center for Cybernetic Applied to Medicine, Havana 11600, Cuba \\ ${ }^{3}$ Cybernetic Center Applied to Medicine, Havana 11600, Cuba \\ Correspondence should be addressed to Zurina Lestayo O’Farrill; zurina@infomed.sld.cu
}

Received 24 October 2012; Accepted 26 November 2012

Academic Editors: P. E. Cattan, Q. Chen, and R. Pereira

Copyright (C) 2013 Zurina Lestayo O'Farrill et al. This is an open access article distributed under the Creative Commons Attribution License, which permits unrestricted use, distribution, and reproduction in any medium, provided the original work is properly cited.

\begin{abstract}
The etiology of Guillain Barré Syndrome (GBS) is not fully clarified, and there is a lack of agreement concerning its putative epidemic character. The low incidence rate of this disease is a disadvantage for employing the traditional statistical methods used in the analysis of epidemics. The objective of this paper is to clarify the GBS epidemic behavior applying a nonlinear time series identification approach. The authors obtained one time series of GBS and nine series of classical infectious epidemics (5 national and 4 international). These data were processed with advanced techniques of statistical time series analysis. This paper shows that GBS behaves similar to the other time series of classical epidemic studied. It corresponds to a nonlinear dynamics, with a point attractor. The spectral analysis pointed to an annual periodicity, and preference for the warmest month of the year was found. These results might suggest that Guillain Barré Syndrome has an epidemic behavior. The adequacy of nonlinear methods for analyzing the dynamics of epidemics, particularly those with low incidence rate, such as GBS was revealed.
\end{abstract}

\section{Introduction}

The Guillain Barré Syndrome is an acute autoimmune neuropathy. It's epidemiology is a broadly approached topic in the literature, but there are still aspects where opinions diverge or are even diametrically opposed [1]. The epidemiological behavior of GBS is one of the most controversial issues. Some authors report outbreaks or "striking fluctuations" in the incidence and confirm that it behaves periodically and shows seasonal variation [2-11]. The occurrence of outbreaks and the presence of periodicity and seasonal preference of the incidence are aspects that suggest an epidemic behavior. Nevertheless, some authors have found that incidence of this syndrome is stable throughout the year and even for longer periods of observation and deny any seasonal variation or periodicity [12-17]. The endemic channel and other statistical tools for epidemiological surveillance show limitations when the epidemic analyzed has a low level of endemicity or when fluctuations of the incidence, which could be considered as outbreaks are not numerically large, as occuring in GBS. Some models have been proposed to study the dynamics of epidemics, but these techniques cannot adequately describe behavior of a series with more complex dynamics [18-21]. The modeling of "biological systems" through nonlinear mathematics ("Chaos theory") has proven to be useful in the understanding of complexity and especially in epidemics [22-36]. However, we have not found in the literature reports which study the behavior of GBS applying a nonlinear identification approach. In this paper nonlinear mathematic tools were applied, for the first time, to analyze GBS time series data. In particular, we have addressed the question whether GBS behaves like an epidemic. The authors also analyzed other nine time series of classical infectious epidemics to compare and determine possible causal links between these ones and GBS.

\section{Materials and Methods}

2.1. Material. Four hundred clinical records of patients discharged from the Institute of Neurology and Neurosurgery of Cuba with the clinical diagnosis of GBS, from 1967 to 1997 , were reviewed. 


\subsection{Methods}

2.2.1. Procedures and Techniques for the Collection of Information. The GBS time series was obtained from patients' admission dates (only data available in a sufficient number of observations). Five time series of national epidemics were also constructed: acute diarrheal diseases (ADD), acute respiratory diseases (ARD), typhoid fever, and mononucleosis and scarlet fever (series available in a sufficient number of observations). This information was provided by the Statistics Department of the Ministry of Public Health. Additionally four time series of international epidemics were also included. The series of measles in New York and Baltimore and mumps in USA were provided by Thomas Schriber MD from Whupertal University (Germany). The series of cholera in the city of Calcutta was taken from the classical book "Health Statistics" [37].

2.2.2. Data Analysis. The databases were created as open office spreadsheets and saved as ASCII files. Advanced statistical techniques of time series analysis were applied and also systems for nonlinear time series analysis developed at the Cybernetics Centre Applied to Medicine.

\subsubsection{Method Used to Determine Whether GBS Was a Random} Independent Process. Data were represented as an intervals' histogram between successive events and were plotted in semilogarithmic coordinates. In this analysis, if dependence is linear (exponential distribution), a random mechanism is suggested.

2.2.4. Linear Identification Method. Linear correlation between the present and the past values, based on the estimation of the lineal correlation coefficient in a multiple regression, model was employed.

2.2.5. Nonlinear Identification Method. The time series is considered as dynamically emerging from a system of the type

$$
x_{t}=f\left(X_{t-1}\right)+e_{t},
$$

where $X_{t-1}=\left(x_{t-1}, x_{t-2}, \ldots, x_{t-m}\right)^{t}$ is a state vector; $f$ : $R^{m} \rightarrow R$ is a smooth map which has been designated as the skeleton of the underlying dynamical system [35]; $\left\{e_{t}, t=\right.$ $1,2, \ldots, N\}$ is a sequence of dynamical noise such that $e_{t}$ is independent of $x_{t-s}$ for $s>0$; and $\mathrm{m}$ is a positive integer, so-called lag. In our case, " $x$ " refers to time intervals elapsed between two successive appearances of cases.

In the most general case, when very few assumptions are made about $f$ (smooth, continuous, stationary) it is convenient to estimate the nonlinear autoregressive function $f$ via a Naradaya-Watson nonparametric kernel [35]. In kernel nonparametric estimation, the estimate of $f$ in (1) at a point $\left(z_{t-1}, z_{t-2}, \ldots, z_{t-n}\right)$ of the state space is obtained as a weighted average of all the observed data $\left(x_{1}, x_{2}, \ldots, x_{n}\right)$. Specifically:

$$
\widehat{f}\left(z_{t-1}, z_{t-2}, \ldots, z_{t-m}\right)=\frac{\sum_{i=m+1}^{N} x_{i} \prod_{j=1}^{m} K\left(\left|z_{t-j}-x_{i-j}\right| / h\right)}{\sum_{i=m+1}^{N} \prod_{j=1}^{m} K\left(\left|z_{t-j}-x_{i-j}\right| / h\right)} .
$$

Here, $K(|z-x| / h)$ is the kernel function and the parameter $h$ is the bandwidth parameter. In our implementation, $h$ is selected via cross validation error minimization.

In this study the following exponential kernel was selected: $k(u)=(1 / 2) e^{-|u|}$.

To find the bandwidth parameter $h$, a cross validation one-step-ahead criterion was applied.

2.2.6. Viewing Attractors. Once the function " $f$ " was estimated, a noise-free realization was obtained by recursively calculating the next value of " $f$ " from the past. As initial values a real segment of the signal was taken. After several hundreds of recursive estimations a series was generated that, by construction, contained no stochastic influences and allowed to detect the presence of a nompoint attractor. In such cases, the series was considered as nonlinear, but in the cases in which there was a point attractor (such as our series of GBS), a complementary approach to demonstrate nonlinearity was necessary. In these situations, we estimated correlation dimension.

2.2.7. Correlation Dimension. The correlation dimension corresponding to the original series was estimated as well as to one generated from a linear autoregressive model estimated from the same original data. A nonlinearity criterion was considered if the value of the dimension of the original series was less than the surrogate one.

2.2.8. Programs. For the nonparametric estimation a program designed in SciLab by the bioinformatics group of CECAM was developed. The SciLab system was also used for plotting of original and generated series. To calculate the correlation dimension the MTRCHAOS program, developed by Michael T. Rosenstein in 1993, was used.

\section{Results and Discussion}

\subsection{Dynamic of Guillain Barré Syndrome}

3.1.1. To Define If GBS Behaved as a Random or Deterministic System. Graphic Representation of the GBS Time's Series. The time series of monthly incidence of the disease is represented in Figure 1. Simple observation of the data might suggest that GBS has an epidemic behavior showing spikes in certain months, where the incidence is higher (It could correspond to outbreaks). On the other hand, in another month there were no cases. Verification of peaks in time's series of typical epidemics is a graphic evidence of the irrefutable existence of an outbreak, but in the case of GBS, where the daily and outbreak incidence is very low, we found this criterion unreliable to assert the epidemic behavior of any disease. 


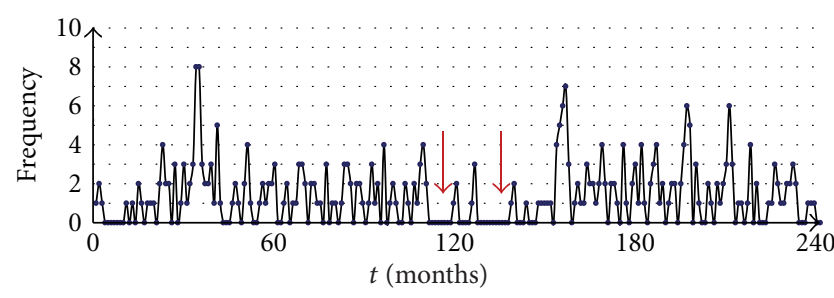

FIGURE 1: Original time series of GBS. The arrows indicate periods where the incidence was zero.

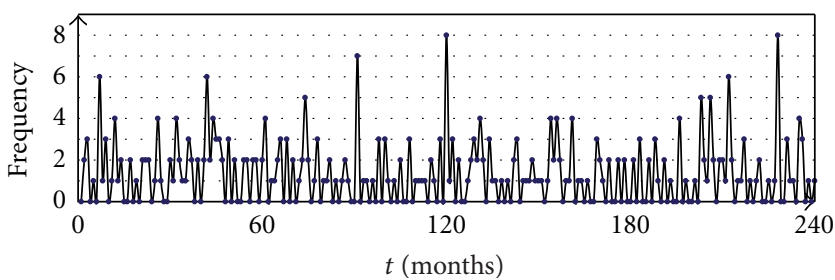

FIgURE 2: Surrogate of GBS time series.

Figure 2 that shows the graphical representation of an artificial series obtained randomizing the original one. This technique makes random and original series comparable. We can see that in the random series there are also peaks that could suggest the presence of outbreaks; even in the spectrum of both series there were no great differences, but a closer look at both figures shows that in the surrogate series the values are regularly distributed throughout each month, while in the original one there are periods of several months where there are no cases (arrows) and others where the incidence is more constant. This aspect might suggest that this is not a random process and has a structure or laws that govern the behavior, but this small detail is not enough.

Testing the GBS Time Series for Randomness. A histogram was constructed for the distribution of intervals between two successive admissions. The $x$-axis contains the logarithms of the intervals and the $y$-axis contains the frequency of appearance of an interval with this size or higher (Figure 3). The graphic reveals that the data are not distributed exponentially, the dependence is not linear, and therefore it is not a random process. This is the first evidence of structure in this data; it behaves (at least partly) as a deterministic system. The next step was then to determine whether the system is linear or nonlinear.

3.1.2. Testing the GBS Time Series for Linearity. Linear Correlation in GBS. The correlation of GBS with their previous values was estimated. The linear autoregressive model had a correlation of 0.45 , which was significant for the number of cases studied $(P<0.01)$. Thus, we concluded that GBS presents correlations with the past.

\subsubsection{Testing the GBS Time Series for NonLinearity. Correla-} tion Dimension. The correlation dimension of the GBS time series was 5.82, a relatively low value. At the same time, the dimension of correlation of the randomized data set was

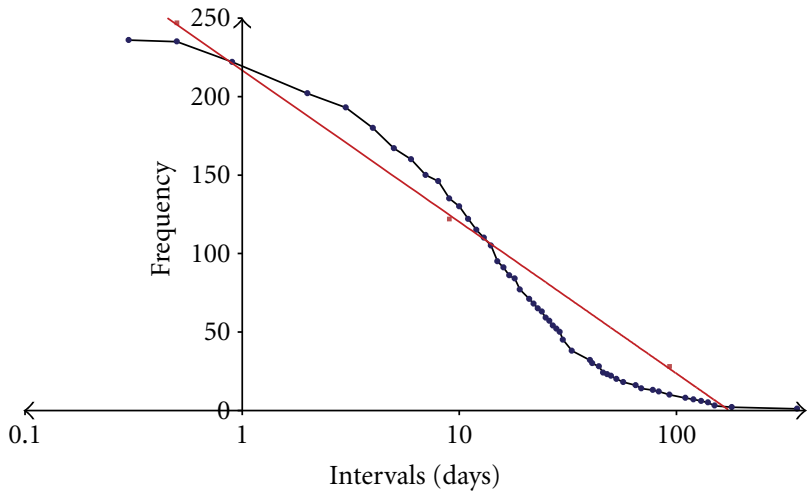

FIGURE 3: Semilog representation of GBS intervals' series: histogram for the distribution of the interval between two successive incomes. Note that the data have no linear dependence.

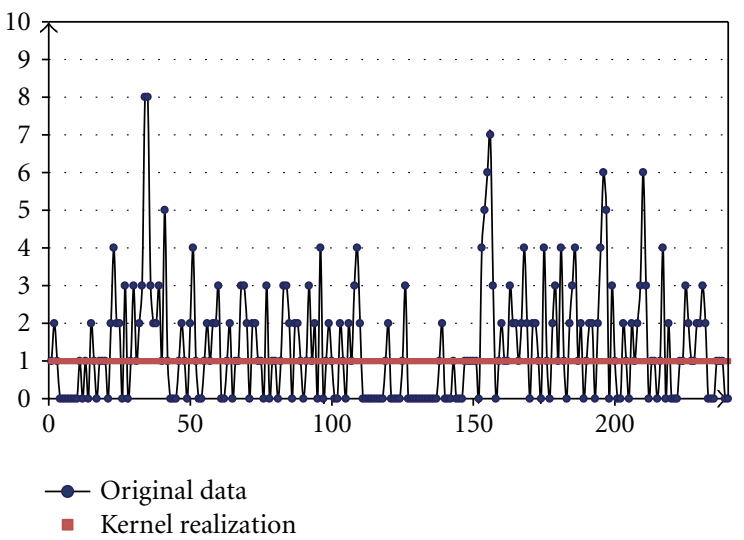

FIGURE 4: Identification of GBS attractor. The Noise-free realization obtained from kernel nonparametric autorregression is a flat line, corresponding to a flat attractor.

8.087. It supports the presence of a nonlinear structure in the GBSs' time series.

Correlation dimension is one of the fundamental concepts of nonlinear dynamics and "chaos theory". In a space reconstruction following the Takens algorithm, correlation dimension appears as one of the dynamical systems invariants. In general it is regarded as a measure of complexity, since it is related to the minimal number of variables required to describe a given system [38].

Estimation of the Attractor. The nonlinear identification method showed the presence of a point attractor. So far, we could identify that GBS behaves as a deterministic, nonlinear system with a point attractor (Figure 4).

According to Philippe, the estimation of the attractor and the spectral analysis are useful tools to detect chaos in time series data [26]. In this paper we tested the usefulness of this method to determine the existence of a deterministic component.

This analysis supports that GBS behaves mathematically as a nonlinear deterministic system. This kind of behavior was also found in other typical infectious epidemics studied 


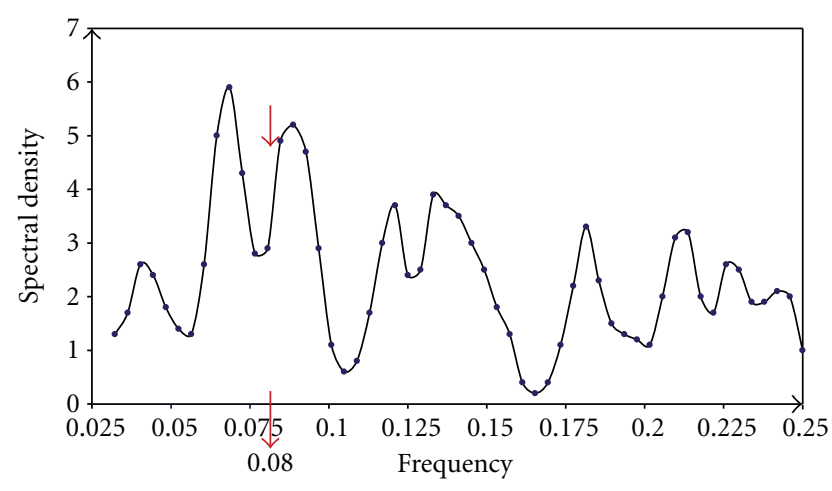

FIGURE 5: Spectrum of the GBS time series. Note that the frequency of 0.08 (corresponding to a 12 -month period) is flanked by the highest peaks in the spectrum.

in this paper: USA mumps, measles in Baltimore, New York measles, cholera in Calcutta, and national series of ADD. Stones analyzed a series of measles in New York for the presence of CAOS and confirmed a low-dimensional chaotic dynamic [32]. Olsen et al. reported low-dimension Chaos in epidemics of mumps, measles, and rubella [34].

\subsection{Periodicity in the Guillain Barré Syndrome}

3.2.1. Detection of Periodicity in the Time Series of GBS. The power spectrum of the signal reveals two peaks corresponding to 11 and 14 months. We assume that these two local peaks are indeed part of a common peak at 12 months period (annual periodicity). The fact is that many epidemics have a year cycles and curiously GBS seems to share also this property of classical epidemics (Figure 5).

Possible Dependence of the Periodicity with the Seasons. Data were averaged by month (for 30 years), and it was observed that in August incidence was almost doubled (Figure 6).

The incidence in our study varies throughout the year but with greater preference for the month of August (one of the warmest months of the year). This is possibly related to environmental factors. The seasonal variation in the incidence in GBS has been analyzed by several researchers $[1,2$, $12,13,16,39-41]$.

3.3. Dynamics of Typical Epidemics. The series of mumps in the USA can be modeled as a nonlinear stochastic system with a chaotic attractor. The measles series in Baltimore and New York have a chaotic attractor too. The series of cholera in Calcutta can be modeled as a limit cycle perturbed by noise. The national Cuban series of acute diarrheal diseases (ADD) has a change of dynamics in the vicinity of the month 150 . The first part of the series shows a chaotic dynamics and the second part of the series, a limit cycle. The national series of acute respiratory disease (ARD) corresponds to a stochastic system with a point attractor. National series of typhoid fever shows a point attractor. National series of mononucleosis and

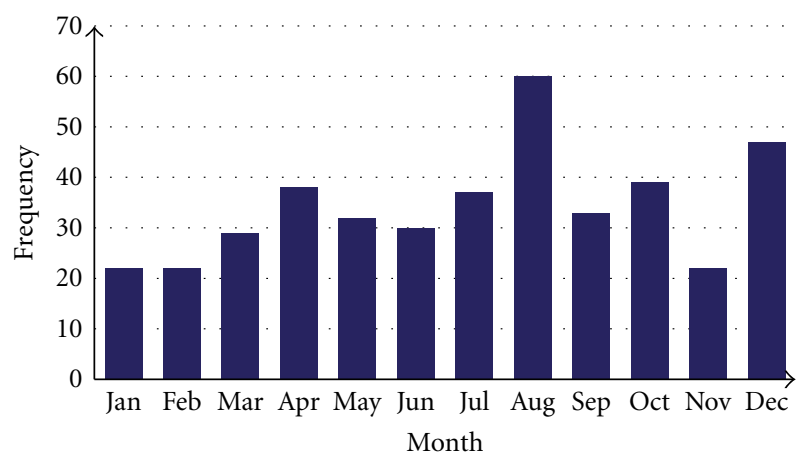

FIgUre 6: Monthly incidence of the GBS. Note that the incidence is higher in August.

scarlet fever were associated with a point attractor perturbed by noise.

\section{Conclusion}

The random nature of GBS was discarded. It can be modeled as a nonlinear deterministic system with a point attractor. This behavior is frequently observed in other typical epidemics. There is an annual periodicity in the GBS series with greater incidence in August.

In this work we applied a nontraditional mathematical method, to clarify some aspects of GBS in which some researchers disagree. Apparently, this is the first attempt in the literature to apply a nonlinear identification technique to a broad group of epidemics datasets and in particular the GBS. We consider that this approach adds additional evidence in favor of the epidemic behavior of this polemic syndrome.

\section{References}

[1] Z. Lestayo-O’Farrill and J. L. Hernández-Cáceres, "Analysis of the behaviour of Guillain-Barre syndrome. Agreements and discrepancies," Revista de Neurologia, vol. 46, no. 4, pp. 230-237, 2008.

[2] J. R. Estrada, O. Hernández, and J. Galarraga, Polirradiculoneuritis Aguda de Causa Desconocida, Ciencia y Técnica, La Habana, Cuba, 1976.

[3] P. L. Hung, W. N. Chang, L. T. Huang et al., "A clinical and electrophysiologic survey of childhood Guillain-Barré syndrome," Pediatric Neurology, vol. 30, no. 2, pp. 86-91, 2004.

[4] J. I. Cuadrado, J. De Pedro-Cuesta, J. R. Ara et al., "GuillainBarré syndrome in Spain, 1985-1997: epidemiological and public health views," European Neurology, vol. 46, no. 2, pp. 83-91, 2001.

[5] E. Chroni, S. Papapetropoulos, G. Gioldasis, J. Ellul, N. Diamadopoulos, and T. Papapetropoulos, "Guillain-Barré syndrome in Greece: seasonality and other clinico-epidemiological features," European Journal of Neurology, vol. 11, no. 6, pp. 383-388, 2004.

[6] M. S. G. Rocha, S. M. D. Brucki, AA de S. Carvalho et al., "Epidemiologic features of Guillain-Barré syndrome in São Paulo, Brazil," Arquivos de Neuro-Psiquiatria, vol. 62, no. 1, pp. 33-37, 2004. 
[7] R. C. Potter and J. B. Kaneene, "A descriptive study of GuillainBarré syndrome in high and low Campylobacter jejuni incidence regions of Michigan: 1992-1999," Neuroepidemiology, vol. 22, no. 4, pp. 245-248, 2003.

[8] R. K. Lyu, L. M. Tang, S. Y. Cheng, W. C. Hsu, and S. T. Chen, "Guillain-Barré syndrome in Taiwan: a clinical study of 167 patients," Journal of Neurology Neurosurgery and Psychiatry, vol. 63, no. 4, pp. 494-500, 1997.

[9] G. M. McKhann, D. R. Cornblath, J. W. Griffin et al., "Acute motor axonal neuropathy: a frequent cause of acute flaccid paralysis in China," Annals of Neurology, vol. 33, no. 4, pp. 333-342, 1993.

[10] T. W. Ho, B. Mishu, C. Y. Li et al., "Guillain-Barre syndrome in northern China. Relationship to Campylobacter jejuni infection and anti-glycolipid antibodies," Brain, vol. 118, part 3, pp. 597-605, 1995.

[11] M. Barzegar, S. Dastgiri, M. H. Karegarmaher, and A. Varshochiani, "Epidemiology of childhood Guillan-Barre syndrome in the north west of Iran," BMC Neurology, vol. 7, article no. 22, 2007.

[12] J. R. Estrada, A. Goyenechea, and C. Herrera, "Brote de polirradiculoneuritis aguda tipo Landry-Guillain-Barré-Ströhl durante una epidemia de dengue," Revista Cubana de Higiene y Epidemiología, vol. 19, pp. 252-265, 1981.

[13] M. J. Sedano, J. Calleja, E. Canga, and J. Berciano, "GuillainBarre syndrome in Cantabria, Spain. An epidemiological and clinical study," Acta Neurologica Scandinavica, vol. 89, no. 4, pp. 287-292, 1994.

[14] Q. Cheng, G. X. Jiang, S. Fredrikson et al., "Epidemiological surveillance of Guillain-Barré syndrome in Sweden, 1996-1997. Network members of the Swedish GBS Epidemiology Study Group," Acta Neurologica Scandinavica, vol. 101, no. 2, pp. 104-110, 2000.

[15] R. D'Alessandro, "A prospective study on the incidence and prognosis of Guillain-Barre syndrome in Emilia-Romagna region, Italy (1992-1993)," Neurology, vol. 48, no. 1, pp. 214-221, 1997.

[16] E. Paolino, V. Govoni, M. R. Tola, I. Casetta, and E. Granieri, "Incidence of the Guillain-Barre syndrome in Ferrara, Northern Italy, 1981-1987," Neuroepidemiology, vol. 10, no. 3, pp. 105-111, 1991.

[17] M. McLean, P. Duclos, P. Jacob, and P. Humphreys, "Incidence of Guillain-Barre syndrome in Ontario and Quebec, 1983-1989, using hospital service databases," Epidemiology, vol. 5, no. 4, pp. 443-448, 1994.

[18] T. Smieszek, "A mechanistic model of infection: why duration and intensity of contacts should be included in models of disease spread," Theoretical Biology and Medical Modelling, vol. 6, no. 1, article no. 25, 2009.

[19] F. C. Diego and G.-R. Gisela, "Variable effect of co-infection on the HIV infectivity: within-host dynamics and epidemiological significance," Theoretical Biology and Medical Modelling, vol. 9, article 9, 2012.

[20] B. Hanley, "An object simulation model for modeling hypothetical disease epidemics-EpiFlex," Theoretical Biology and Medical Modelling, vol. 3, article no. 32, 2006.

[21] G. Sertsou, N. Wilson, M. Baker, P. Nelson, and M. G. Roberts, "Key transmission parameters of an institutional outbreak during the 1918 influenza pandemic estimated by mathematical modelling," Theoretical Biology and Medical Modelling, vol. 3, article no. 38, 2006.
[22] S. M. . Blower, "Book Reviews (epidemic models: their structure and relation to data)," American Journal of Epidemiology, vol. 143, no. 10, 1996.

[23] P. Villoslada, L. Steinman, and S. E. Baranzini, "Systems biology and its application to the understanding of neurological diseases," Annals of Neurology, vol. 65, no. 2, pp. 124-139, 2009.

[24] F. F. Orsucci, "The paradigm of complexity in clinical neurocognitive science," Neuroscientist, vol. 12, no. 5, pp. 390-397, 2006.

[25] M. Tabatabai, D. K. Williams, and Z. Bursac, "Hyperbolastic growth models: theory and application," Theoretical Biology and Medical Modelling, vol. 2, article 14, 2005.

[26] P. Philippe, "Chaos, population biology, and epidemiology: some research implications," Human Biology, vol. 65, no. 4, pp. 525-546, 1993.

[27] L. Billings and I. B. Schwartz, "Exciting chaos with noise: unexpected dynamics in epidemic outbreaks," Journal of Mathematical Biology, vol. 44, no. 1, pp. 31-48, 2002.

[28] S. R. Duncan, S. Scott, and C. J. Duncan, "Modelling the different smallpox epidemics in England," Philosophical Transactions of the Royal Society B, vol. 346, no. 1318, pp. 407-419, 1994.

[29] M. G. M. Gomes, A. Margheri, G. F. Medley, and C. Rebelo, "Dynamical behaviour of epidemiological models with suboptimal immunity and nonlinear incidence," Journal of Mathematical Biology, vol. 51, no. 4, pp. 414-430, 2005.

[30] A. Korobeinikov, "Global properties of infectious disease models with nonlinear incidence," Bulletin of Mathematical Biology, vol. 69, no. 6, pp. 1871-1886, 2007.

[31] S. M. Moghadas and M. E. Alexander, "Bifurcations of an epidemic model with non-linear incidence and infectiondependent removal rate," Mathematical Medicine and Biology, vol. 23, no. 3, pp. 231-254, 2006.

[32] L. Stone, "Coloured noise or low-dimensional chaos?" Proceedings of the Royal Society B, vol. 250, no. 1327, pp. 77-81, 1992.

[33] J. E. Truscott and C. A. Gilligan, "Response of a deterministic epidemiological system to a stochastically varying environment," Proceedings of the National Academy of Sciences of the United States of America, vol. 100, no. 15, pp. 9067-9072, 2003.

[34] L. F. Olsen, G. L. Truty, and W. M. Schaffer, "Oscillations and chaos in epidemics: a nonlinear dynamic study of six childhood diseases in Copenhagen, Denmark," Theoretical Population Biology, vol. 33, no. 3, pp. 344-370, 1988.

[35] B. Cheng and H. Tong, "On consistent nonparametric order determination and chaos," Journal of the Royal Statistical Society $B$, vol. 54, no. 2, pp. 427-449, 1992.

[36] B. Bolker, "Chaos and complexity in measles models: a comparative numerical study," IMA Journal of Mathematics Applied in Medicine and Biology, vol. 10, no. 2, pp. 83-95, 1993.

[37] U. J. Barret, Estadísticas Sanitarias, Edición Revolucionaria, La Habana, Cuba, 1964.

[38] P. Grassberger and I. Procaccia, "Measuring the strangeness of strange attractors," Physica D, vol. 9, no. 1-2, pp. 189-208, 1983.

[39] L. Valenciano, E. Nájera, F. P. Gallardo, A. González, and J. Enrech, "Outbreak of paralytic illness of unknown etiology in albacete, Spain," American Journal of Epidemiology, vol. 94, no. 5, pp. 450-456, 1971.

[40] C. J. Coe, "Guillain-Barre syndrome in Korean children," Yonsei Medical Journal, vol. 30, no. 1, pp. 81-87, 1989.

[41] Y. Aladro-Benito, M. A. Conde-Sendin, C. Muñoz-Fernández et al., "Guillain-Barré syndrome in the northern area of Gran Canaria and the island of Lanzarote," Revista de Neurología, vol. 35, no. 8, pp. 705-710, 2002. 


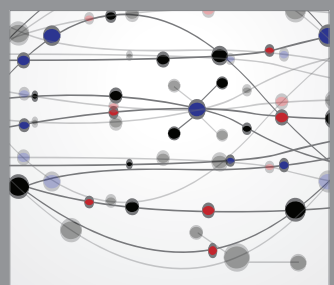

The Scientific World Journal
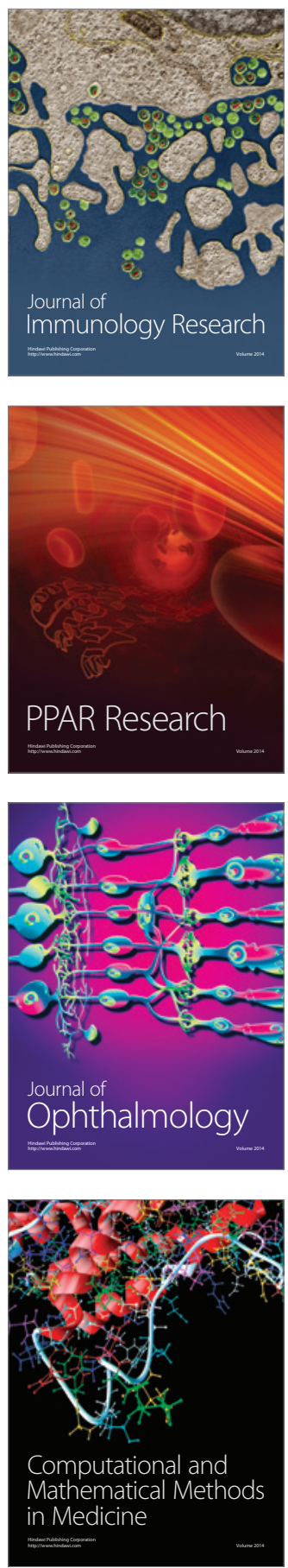

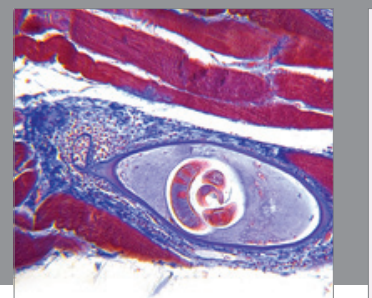

Gastroenterology

Research and Practice
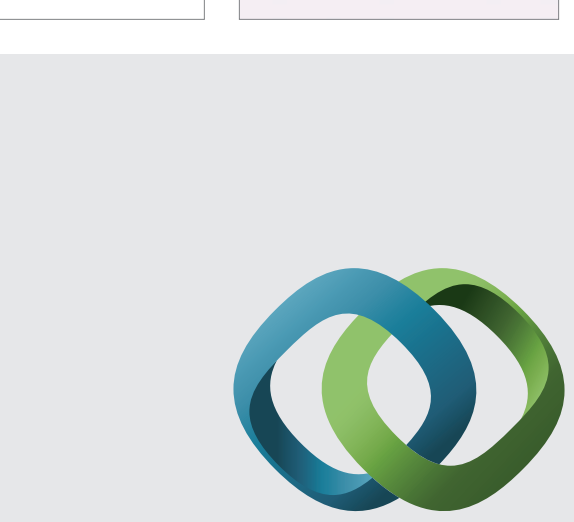

\section{Hindawi}

Submit your manuscripts at

http://www.hindawi.com
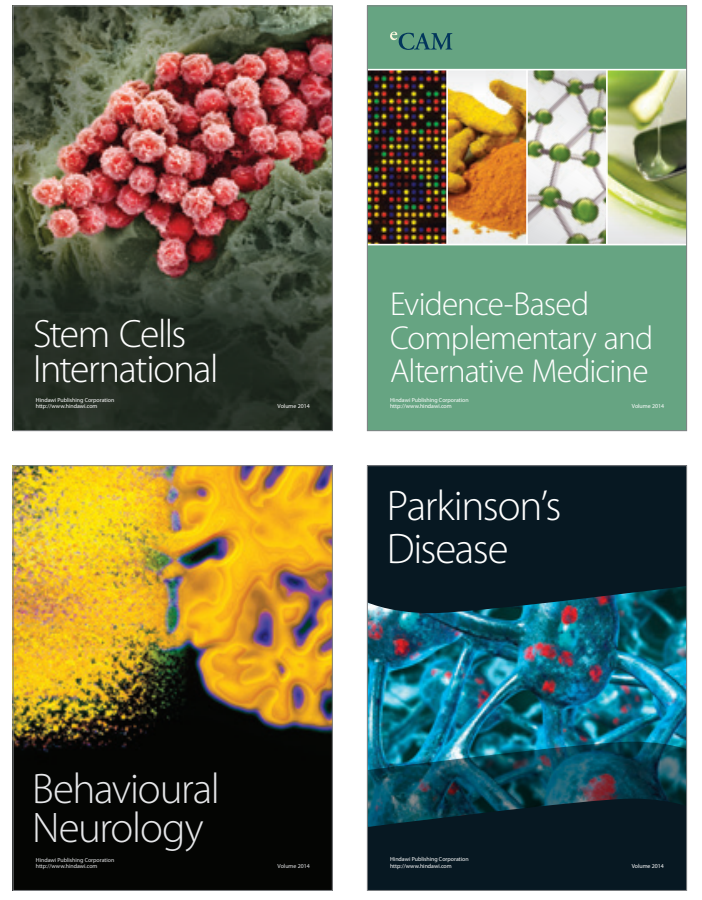
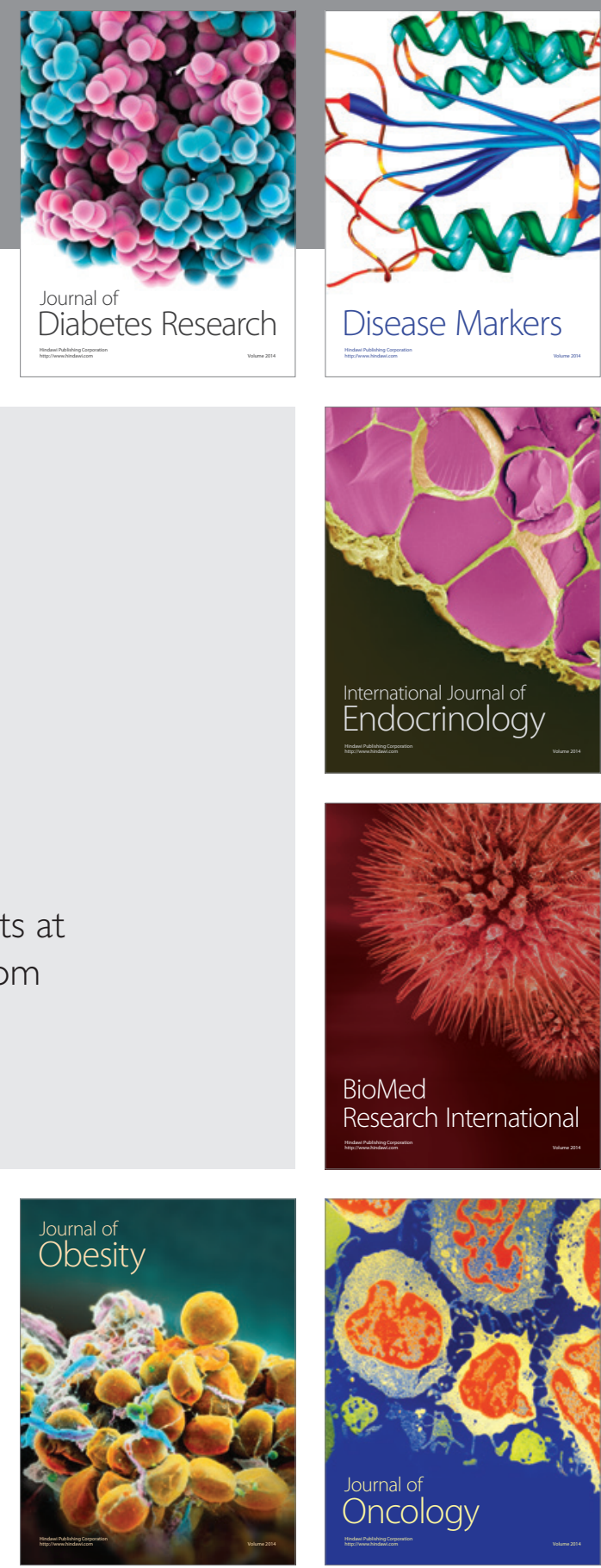

Disease Markers
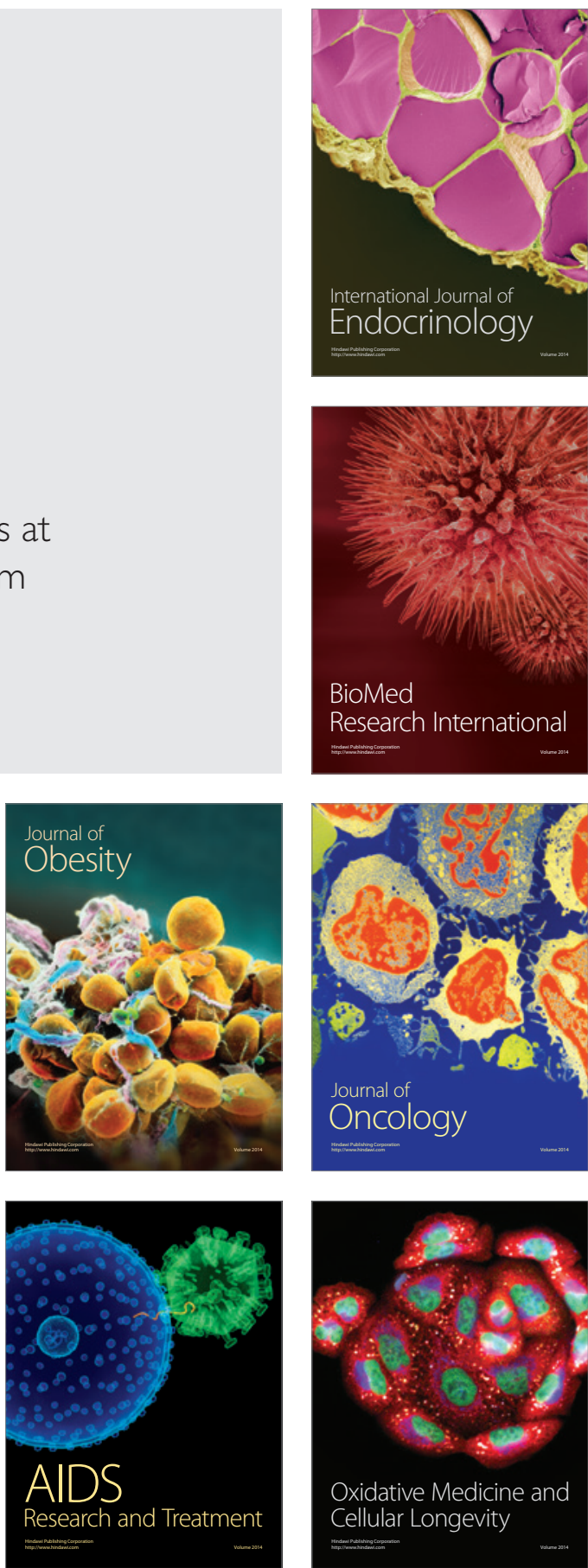